\title{
The Missing M Dwarfs
}

\author{
Richard F. Jameson, Paul D. Dobbie \\ Dept. of Physics 8 Astronomy, University of Leicester, Leicester, UK.
}

David J. Pinfield

Astrophysics Research Institute, Liverpool John Moores University, Birkenhead, UK

Simon T. Hodgkin

Institute of Astronomy, University of Cambridge, Cambridge, UK

\begin{abstract}
We present evidence for a drop in the luminosity function around spectral type M7-M8. This shows in both field stars and the clusters $\sigma$-Orionis, the Trapezium, IC 348, IC 2391, $\alpha$-Perseii, the Pleiades and, possibly, the globular cluster M4. This can be explained by a sudden drop in the luminosity-mass relation. Theoretical models, which include dust, do not show this effect, although they predict dust forming at about spectral type M7. We suggest that this drop in the luminosity-mass relation could be modelled by considering larger size dust grains. The drop in the luminosity-mass relation suggests that star/brown dwarf masses below M7-M8 will be larger than predicted by current models.
\end{abstract}

\section{Introduction and evidence for the missing $M$ dwarfs}

The detailed shape of the luminosity function (LF) of a star cluster or the field stars provides information on the luminosity-mass relation of the stars or brown dwarfs in the cluster/field. This is because the LF can be written in terms of the mass function and the magnitude-mass relation, see below. A good example is the Wielen dip at $M_{V}=7$ (Wielen, Jahreiss \& Kruger 1983). This has been attributed to a steepening of the luminosity-mass due to strong $\mathrm{H}$ - opacity in atmospheres of these stars (Kroupa et al. 1990). In this paper we discuss the evidence for and consequences of a similar dip in the LF around spectral type M7-M8.

In our recent paper on brown dwarfs in the Pleiades (Jameson et al. 2002) we realised that there is a deficit of brown dwarf Pleiads with apparent magnitudes $I_{\mathrm{C}}=18.15-18.65$, corresponding to an $I-K \approx 3.50-3.75$ or a spectral type of M7-M8. The relevant colour magnitude diagram is shown in Figure 1. We have excluded from the LF clear binaries since their component luminosities/masses are uncertain. There are $2 \pm 1.4$ brown dwarfs in the gap. Extrapolating across the top of the gap would suggest $9 \pm 3$ brown dwarfs, errors from random number statistics. The LF dip is clearly real at an approximately 

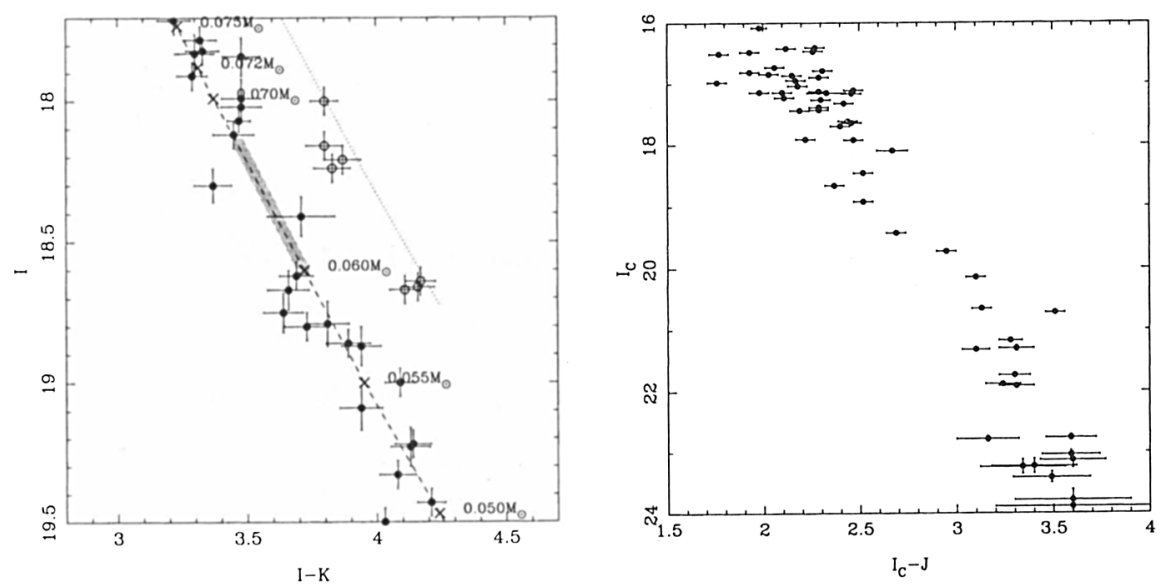

Figure 1. H-R diagrams for the Pleiades(left) and $\sigma$-Orionis(right) showing the gaps at M7-M8.

$90 \%$ confidence level. Our data also show that the width of the gap is smaller at longer wavelengths. The gap width being $0.50,0.35,0.30$ and 0.25 magnitudes at $I, J, H$ and $K$ respectively. The width of the gap is thus probably $\sim 0.3$ bolometric magnitudes.

Searching the literature we rapidly realised that there is evidence for a similar dip in a number of other clusters. The statistical significance of these gaps is not always as good as in the well studied Pleiades, but given that the gap is found in the field, six open clusters and possibly the globular cluster M4, we believe this luminosity dip is universal. Figure 1 also shows the HR diagram for the $\sigma$-Orionis cluster using the data from Béjar et al. (2001). The gap extends from $I=19-20$ or $2.6<I-J<3.0$ again corresponding to spectral type M7/8. Note in this young cluster $(\sim 3 \mathrm{Myr})$ the gap is bigger $(1.1 \mathrm{mag})$ in $I$. This is because the luminosity-mass relation is steeper in young clusters, both $\mathrm{T}_{\mathrm{eff}}$ and radius are decreasing with decreasing mass. In more mature clusters the radius is approximately constant. Lucas \& Roche (2000) have surveyed the Trapezium cluster for brown dwarfs. Again the gap is very evident in the two colour diagram of the cluster at $2.6<I-J<2.8$, their Figure 3. We think the slightly narrower gap in this young cluster is due to binaries being pushed into the $I-J \sim 2.9$ region by their dereddening procedure. Muench et al. (2002) also find this dip in the LF of the Trapezium cluster. Luhman (1999) hints at a deficit of brown dwarfs in IC 348 between spectral types M7 to M8. Barrado y Navascués et al. (2001) present data on the cluster IC 2391. Again the gap is evident, albeit at a rather low statistical significance. Stauffer et al. (1999) have surveyed the $\alpha$-Per cluster and also find the dip in the $\mathrm{LF}$ at $\mathrm{M}_{I} \sim 12.5$, again spectral type M7-M8.

Turning to older objects, Reid \& Cruz (2002) have assembled data on field stars within 20 pc of the Sun. Their Figure 11 shows the gap at $2.6<I-J<3.0$. Finally, Richer et al. (2002) have used the HST to make an excellent proper 

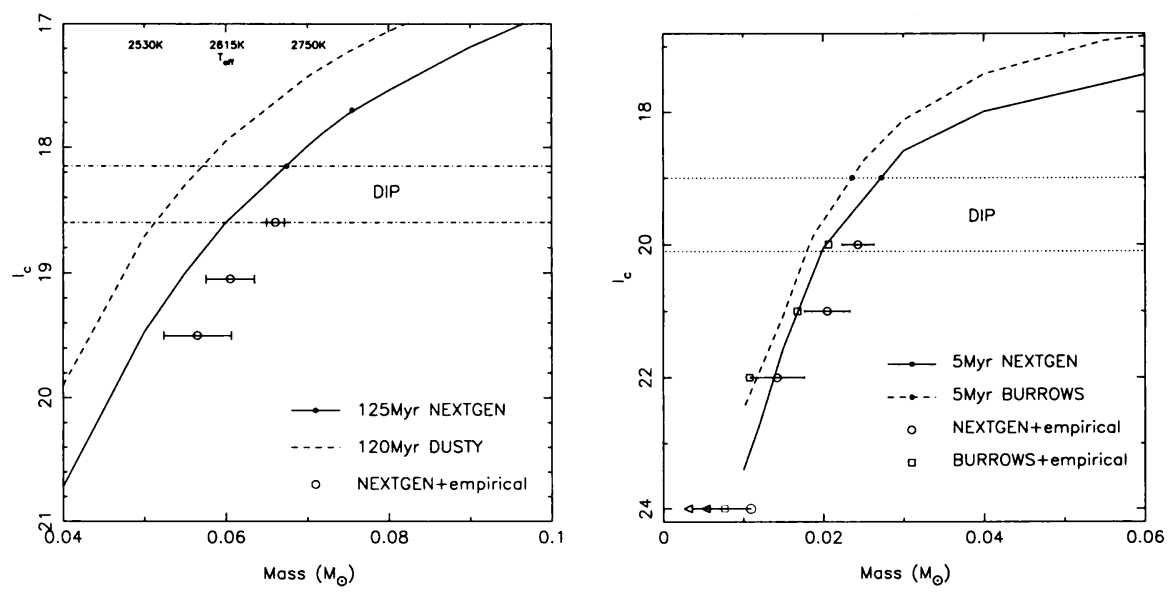

Figure 2. Magnitude-mass relations for the Pleiades(left) and $\sigma$-Orionis(right).

motion survey of the globular cluster M4. A small gap is evident at low statistical significance at $V=27.7, I=23.7$ and $V-I=4.0$, see their Figure 2.

\section{Masses in and below the gap}

This LF dip occurs over a very wide range of masses as we go from young clusters $(\sim 1 \mathrm{Myr})$ to stars of the globular cluster M4 ( 12 Gyr). This argues against it being due to the mass of the objects. The obvious way to explain the luminosity function dip is by a sharp fall in the luminosity-mass relation. The LF $d N / d M$ is related to the mass function $d N / d m$ by Equation 1

$$
d N / d M=\frac{d N}{d m} / \frac{d M}{d m}
$$

where $m$ is mass and $M$, absolute magnitude. Clearly if $d M / d m$ increases, the LF must fall. Furthermore, if we assume the mass function is a power law, $\mathrm{dN} / \mathrm{dm} \propto \mathrm{m}^{-\alpha}$, then by integrating twice and dividing we have Equation 2,

$$
N_{23} / N_{12}=\left(m_{2}^{1-\alpha}-m_{3}^{1-\alpha}\right) /\left(m_{1}^{1-\alpha}-m_{2}^{1-\alpha}\right)
$$

where $\mathrm{N}_{23}$ is the number of stars between masses $\mathrm{m}_{2}$ and $\mathrm{m}_{3}$ and $\mathrm{N}_{12}$ the number between $m_{1}$ and $m_{2}$. If we trust theoretical models to give $m_{1}$ and $m_{2}$ from their measured absolute magnitudes and we assume a power law index $\alpha=0.4$ for the Pleiades, then we can solve the above equation for $\mathrm{m}_{3}$, since $\mathrm{N}_{12}$ and $\mathrm{N}_{23}$ are measured quantities. In this way we can determine the mass $\mathrm{m}_{3}$ at any absolute magnitude. The result is not very sensitive to $\alpha$ unless $\mathrm{m}_{3} \ll \mathrm{m}_{2}$. Using the NEXTGEN model of Baraffe et al. (1998) to fix $\mathrm{m}_{1}$ and $\mathrm{m}_{2}$ above the gap, we derive the $I$ magnitude-mass relation for the Pleiades, see Figure 2. Here 
we have excluded the obvious binary stars. Including the binaries, assuming they are equal mass binaries and making allowance for binaries missing from the top of Figure 1, gives a broadly similar result. Also shown in Figure 2 is the DUSTY model of Chabrier et al. (2000).

Figure 2 also shows the luminosity-mass relation similarly derived for the $\sigma$-Orionis cluster, using this time both the NEXTGEN model, open circles and also the Burrows et al. (1997) model, squares. In this case single stars/ brown dwarfs and binaries are lumped together since they are not well distinguished in the observed HR diagram. Note that none of the models predict the derived steepening of the luminosity-mass relation. It follows that masses derived from these models should be treated with some caution below the spectral type M7M8.

\section{Discussion}

What is the cause of this steepening of the luminosity-mass relation and why is it not predicted by the theoretical models? Theoretical models predict the onset of dust formation in brown dwarf/stellar atmospheres of spectral type M7, $\mathrm{T}_{\text {eff }} \sim$ $2700 \mathrm{~K}$. There is also observational evidence, Jones \& Tsuji (1997), for this onset of dust formation. This additional source of opacity then provides a natural explanation for the steepening of the luminosity-mass relation. The NEXTGEN models assume dust particle sizes $<0.24 \mu \mathrm{m}$, i.e. Rayleigh scatterers. If the dust particles are larger, i.e. Mie scatterers, the opacity is increased, so we suggest that by increasing the dust particle sizes may enable theoretical models to predict the luminosity dip. Note also that Mie scatterers should have a more pronounced effect at shorter wavelength, as observed. Also low metallicity stars in globular clusters will produce less dust, perhaps explaining the very small gap seen in M4.

\section{References}

Baraffe, I., Chabrier, G., Allard, F., Hauschildt, P.H. 1998, A\&A, 337, 403

Barrado y Navascués, D., et al. 2001, ApJS, 134, 103

Béjar, V.J.S., et al. 2001, ApJ, 556, 830

Burrows, A., et al. 1997, ApJ, 491, 856

Chabrier, G., Baraffe, I., Allard, F., Hauschildt, P. 2000, ApJ, 542, 462

Jameson, R.F., et al. 2002, MNRAS, in press

Jones, H.R.A., Tsuji, T. 1997, ApJL, 480, 39

Kroupa, P., Tout, C.A., Gilmore, G. 1990, MNRAS, 244, 76

Lucas, P.W., Roche, P.F. 2000, MNRAS, 314, 858

Muench, A.A., Lada, E., Lada, C., Alves, J. 2002, ApJ, 573, 366

Reid, I.N., Cruz, K. 2002, AJ, 123, 2806

Richer, H., et al. 2002, ApJL, 574, 151

Stauffer, J.R., et al. 1999, ApJ, 527, 219103

Wielen, R., Jahreiss, H., Kruger, R. 1983, In: Proc. IAU Colloq. 76 